\title{
Blanqueo de caolín por medio de lixiviación en pilas con ácido oxálico
}

\author{
Bleaching kaolin by heap leaching with oxalic acid.
}

\section{Branqueamento de caulim por lixiviação em pilhas com ácido oxálico}

Manuel Julián Barros-Daza ${ }^{1}$, Pablo Bustamante-Baena², Moisés Oswaldo Bustamante-Rúa ${ }^{3}$

Forma de citar: M. J. Barros-Daza, P. Bustamante-Baena, M. O. Bustamante-Rúa, "Blanqueo de caolín por medio de lixiviación en pilas con ácido oxálico", Respuestas, vol. 21, no. 1, pp. 65-76, 2016.

Recibido:

Junio 11 de 2015

Aceptado:

Agosto 27 de 2015

\section{${ }^{1}$ Ingeniero de Minas y Metalurgia} mjbarrosd@unal.edu.co Orcid: 0000-0002-3481-4331

Universidad Nacional de

Colombia

Medellín, Colombia.

${ }^{2}$ Ingeniero de Minas y Metalurgia

pabustamanteba@unal.edu.co Orcid: 0000-0002-8409-5531

Universidad Nacional de Colombia

Medellín, Colombia.

${ }^{3}$ Doctor en Ciencias de la Ingeniería con Mención en Metalurgia Extractiva mobustam@unal.edu.co Orcid: 0000-0002-1692-991X

Universidad Nacional de Colombia Medellín, Colombia.

\section{Resumen}

Se estudiaron los resultados experimentales de lixiviación en pilas para el blanqueo del caolín a escala de laboratorio basados en un desarrollo teórico. El objetivo principal fue evaluar la remoción de óxidos de hierro sobre la superficie del caolín, Método con el uso de diferentes concentraciones de ácido oxálico en un tiempo de 48 días. Resultado. El índice de blancura del caolín de alimento fue del $52.1 \%$, con porcentajes de hierro del $0.19 \%$. A partir de los ensayos experimentales, Conclusión los resultados mostraron que para las concentraciones de $0.15 \mathrm{M}, 0.015 \mathrm{M}$ y $0.95 \mathrm{M}$, las remociones de hierro fueron alrededor del $72.2 \%, 26.7 \%, 90.8 \%$ y los índices de blancura obtenidos fueron de $90.2 \%$, $83.8 \%$ y $97 \%$ respectivamente.

Palabras clave: Acido Oxálico, Blanqueo de Caolín, Hidrometalurgia, Lixiviación en pilas, Óxidos de hierro.

\begin{abstract}
The experimental results of leaching were studied in batteries for bleaching kaolin scale The experimental results of leaching were studied in batteries for bleaching kaolin scale development based on a theoretical laboratory. The main objective was to evaluate the removal of iron oxides on the surface of kaolin, Methods: the use of different concentrations of oxalic acid drip for 48 days in each heap. Results: The whiteness index of feed Kaolin is $52.1 \%$, with an iron content of $0.19 \%$. Conclusions: The results showed that for acidic concentrations $0.15 \mathrm{M}, 0.015 \mathrm{M}$ and $0.95 \mathrm{M}$, the iron removed was approximately $72.2 \%, 26.7 \%, 90.8 \%$ and the whiteness index rose to $90.23 \%, 83.8 \%$, and $97 \%$ respectively.
\end{abstract}

Key words: Oxalic Acid, Bleaching Kaolin, Hydrometallurgy, Heap leaching, Iron Oxides.

\section{Resumo}

Estudaram-se os resultados experimentais de lixiviação por pilha para o branqueamento do caulim a escala de laboratório, baseados num desenvolvimento teórico. Objetivo: 
avaliar a remoção de óxidos de ferro sobre a superfície do caulim. Método: usaram-se diferentes concentrações de ácido oxálico num tempo de 48 dias. Resultado: A partir dos ensaios experimentais se obteve que o índice de brancura do caulim de alimento foi do $52.1 \%$, com porcentagens de ferro do $0.19 \%$. Conclusão: os resultados mostraram que para as concentrações de $0.15 \mathrm{M}, 0.015 \mathrm{M}$ e $0.95 \mathrm{M}$, as remoções de ferro foram em torno de $72.2 \%, 26.7 \%$ e $90.8 \%$ e os índices de branqueamento obtidos foram de $90.2 \%, 83.8 \%$ e $97.0 \%$, respectivamente.

Palavras-chave: Acido Oxálico, Branqueamento do Caulim, Hidrometalurgia, Lixiviação por pilhas, Óxidos de ferro.

\section{Introducción}

Elcaolín es una roca compuestaprincipalmente por minerales del grupo de la caolinita, que presenta una composición química de silicato de aluminio hidratado $\left(\mathrm{Al}_{2} \mathrm{Si}_{2} \mathrm{O}_{5}(\mathrm{OH})_{4}\right)$, originándose debido a la meteorización de rocas feldespáticas y micas presentes en pegmatitas y esquistos micáceos [1].

El caolín juega un papel importante a nivel industrial, ya que presenta múltiples características beneficiosas que mejoran las propiedades de algunos materiales como son: químicamente inerte, buen poder de recubrimiento, poco abrasivo, opacidad cuando se utiliza como pigmento, inodoro, aislante eléctrico, moldeable, no toxico y resistente a altas temperaturas [2].

Algunas aplicaciones industriales donde encontramos la presencia de caolín son: en la fabricación de pinturas (pigmentos de extensión), cerámicos (sanitarios, tejas de alto grado, vajillas, objetos de baños etc.) papel, refractarios, cemento y cosméticos[3].
El índice de blancura es la principal exigencia para la aplicación a nivel industrial de arcillas caoliníticas, convirtiéndose la blancura en un valor agregado. Es por esto que altas reservas de caolín no pueden ser utilizadas para aplicaciones industriales, debido a las limitaciones que presentan en lo que respecta al índice de blancura que poseen. Por ejemplo, en el caso de la fabricación de papel, el caolín requiere una blancura superior al $80 \%$, dependiendo del tipo y calidad del papel. Para la utilización del caolín en cerámicos se exige una blancura del orden del 78-83\% y del 75$80 \%$ en el producto de loza para baño. Para el caso de la fabricación de pinturas se requieren una blancura del $83 \%$ [4].

La causa principal que afecta la blancura del caolín son la presencia de contaminantes adheridos sobre la superficie como: óxidos de hierro (Hematita), Hidróxidos de hierro (goethita-lepidocrocita) y óxidos hidratados de hierro férrico, dándole una coloración al caolín de anaranjada hasta rojiza. Presencia de hasta $0.4 \%$ de hierro férrico en el caolín son suficientes para darle coloración a depósitos caoliniticos [5]. La Tabla I muestra la mineralogía de los principales minerales de fierro ("Fe-baering") en las arcillas que aportan color.

Tabla I. Minerales tipo "Fe-bearing" que aportan color a arcillas.

\begin{tabular}{|c|c|}
\hline Mineral & Estructura \\
\hline Anatasa & $\mathrm{TiO}_{2}$ \\
\hline Goethita & $\mathrm{FeO}(\mathrm{OH})$ \\
\hline Hematita & $\mathrm{Fe}_{2} \mathrm{O}_{3}$ \\
\hline Ilmenita & $\mathrm{FeTiO}_{3}$ \\
\hline Magnesita Titanífera & $\mathrm{Fe}_{3-\mathrm{x}} \mathrm{Ti}_{\mathrm{x}} \mathrm{O}_{4}$ \\
\hline
\end{tabular}

Fuente: [6]

El proceso industrial actual para el blanqueo de caolín consiste en la disolución selectiva del ion férrico $\left(\mathrm{Fe}^{3+}\right)$ en una solución ácida con $\mathrm{pH}$ del orden de 1.0 en (1) y posterior reducción con ditonito de sodio $\left(\mathrm{Na}_{2} \mathrm{~S}_{2} \mathrm{O}_{4}\right)$, como se indica en la (2) y (3) [6]. 


$$
\begin{gathered}
\mathrm{Fe}_{2} \mathrm{O}_{3(\text { sólidb })}+2 \mathrm{H}^{+} \leftrightarrow 2 \mathrm{Fe}_{\mathrm{aq}}^{3+}+2 \mathrm{H}_{2} \mathrm{O} \\
\mathrm{S}_{2} \mathrm{O}_{4}^{2-}+2 \mathrm{H}_{2} \mathrm{O} \leftrightarrow 2 \mathrm{SO}_{3}^{2-}+4 \mathrm{H}^{+}+2 \mathrm{e}^{-} \\
\mathrm{Fe}^{3+}+\mathrm{e}^{-} \leftrightarrow \mathrm{Fe}^{2+}
\end{gathered}
$$

A pesar de las condiciones de acidez y la presencia adecuada de ditonito de sodio, la reacción de reducción del ion férrico / es fuertemente reversible generando un proceso de blanqueo altamente ineficiente [7]. Dicha reversibilidad es asociada a una reacción competencia, producto de la reducción del oxígeno disuelto en el agua en (4) incrementando los potenciales de concentración de hidrosulfito para alcanzar los niveles de blanqueo que industrialmente se requieren [6],[7] .

$$
\mathrm{O}_{2 \text { (Acuoso) }}+2 \mathrm{e}^{-} \rightarrow \mathrm{O}^{2-}
$$

Esta competencia la relacionó [7] con la aparición de precipitados de Fe en forma de sulfatos hidratados de hierro y jarositas que vuelven a dar coloración a los minerales de arcilla que han sido blanqueados.

Además, este proceso genera demasiados riesgos bajo la mirada de las actuales regulaciones ambientales y de desarrollo minero sostenible, por lo que se hace indispensable implementar nuevas técnicas, con menor consumo de agua y energía

Por otro lado, [8] reporta que algunos ácidos débiles forman complejos selectivamente con los iones de Fe, como el caso del ácido oxálico que al combinarse el ion oxalato con el hierro, se forma el oxalato de hierro como complejo en medio acuoso en (5).

$$
\mathrm{Fe}_{2} \mathrm{O}_{3(\mathrm{~s})}+2 \mathrm{HC}_{2} \mathrm{O}_{4(\mathrm{aq})}^{-}+6 \mathrm{H}_{(\mathrm{aq})}^{+} \rightarrow 2\left[\mathrm{Fe}^{3+} \mathrm{HC}_{2} \mathrm{O}_{4}\right]_{(\mathrm{aq})}^{2+}+3 \mathrm{H}_{2} \mathrm{O}_{(\mathrm{l})}
$$

Por lo tanto el presente trabajo experimental tiene como objetivo estudiar y evaluar el blanqueamiento de caolín a través del método de lixiviación en pilas usando acido oxálico en diferentes concentraciones. Dicho estudio y evaluación se centrará en la comparación de los índices de blancura de entrada y salida del caolín. Evidenciando que los resultados de este nuevo proceso reducen las fuertes condiciones acidas de los procesos clásicos y la reversibilidad de la reducción de ion férrico, además disminuye la reacción competencia del oxígeno disuelto en el agua.

\section{Materiales y métodos}

\subsection{Muestra de caolín}

El caolín utilizado es de una mina localizada en el Municipio de La Unión, Antioquia, ubicado aproximadamente a $35 \mathrm{~km}$ del sureste de la ciudad de Medellín [9].
En orden de caracterizar la muestra de caolín, la técnica usada para cuantificar las fases cristalinas fue la de Difracción de Rayos X (DRX) (Siemens, D5000). Para cuantificar la cantidad inicial de hierro en el mineral caoliniticos y posterior caracterización de las soluciones acuosas de descarga, se utilizó el espectrofotómetro de absorción atómica (Thermoscientific, Ice $3000^{\circledR}$ ) equipado con una lámpara de cátodo hueco de hierro. Para la caracterización de la distribución de tamaño de partícula del caolín se utilizó un medidor de distribución de tamaño de partícula tipo laser MasterSize 2,000E ${ }^{\circledR}$.

\subsection{Lixiviación en Pilas ("heap leaching process")} a técnica implementada para menas de bajo tenor ha sido la lixiviación en pilas. Dicha técnica reduce notablemente el consumo 
Vol. 21

No. 1

Enero - Junio 2016

ISSN 0122-820X

E-ISSN 2422-5053

PP: 65-76 de energía y la intensidad de los procesos de separación sólido-líquido en las plantas metalúrgicas [10].

La Figura 1 muestra un esquema del proceso de lixiviación en pilas ("Heap leaching") que en la actualidad se usa para la explotación de minerales de cobre, oro y uranio principalmente. Sin embargo a pesar de las garantías, esta técnica presenta serios inconvenientes cuando las menas poseen altos porcentajes de arcillas, para lo cual se ha desarrollado un pretratamiento con el fin de eliminar este efecto, mediante la aglomeración del mineral.

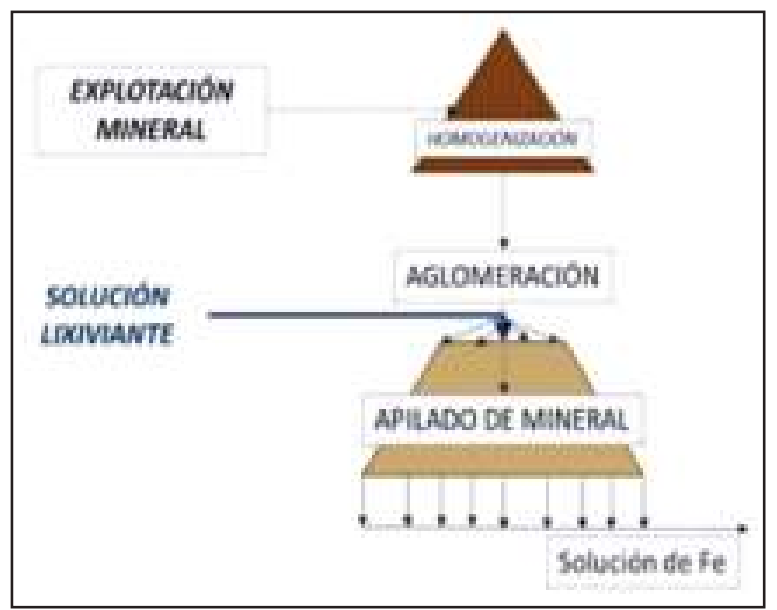

Figura. 1. Esquema de proceso de lixiviación en pilas Fuente: [10]

\subsection{Montaje experimental}

El proceso experimental se llevó a cabo en tubos de acrílico de 1 metro de largo y 0.12 metro de diámetro. Previamente se realizó un pretratamiento del mineral para la producción 68 de glómeros. En la parte superior de los tubos de acrílico se instaló un rociador, que permitió regular el caudal de la solución de Ácido oxálico goteada a la pila del mineral. En la parte inferior de la pila se instaló un dispositivo de descarga que conducía la solución rica en hierro hasta un tanque para su almacenamiento (ver Figura 2).

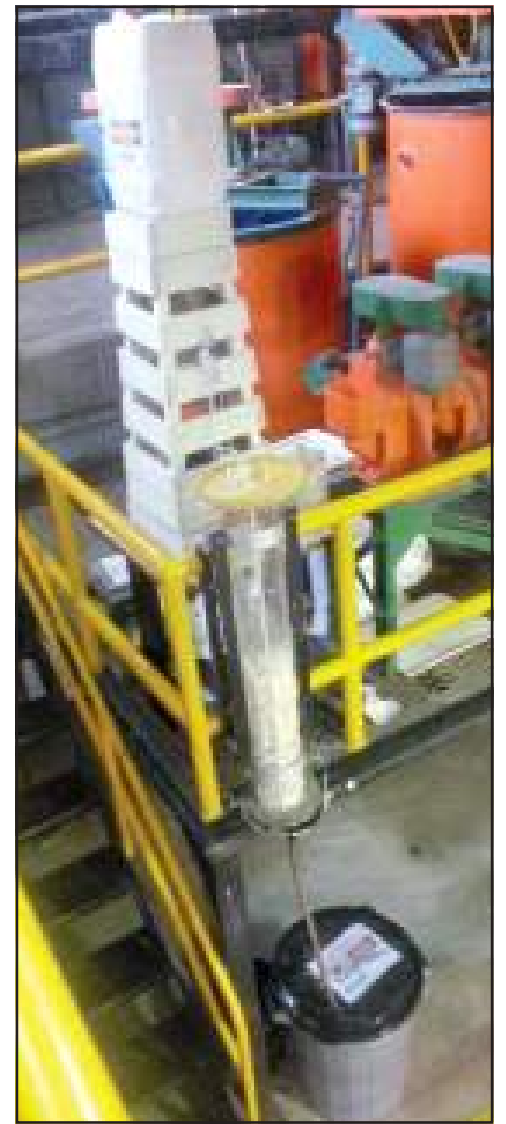

Figura 2. Montaje experimental en laboratorio experimental Fuente: Autor

La concentración del ácido goteada a cada pila se muestra en la Tabla II, durante un tiempo aproximado de 49 días, a presión atmosférica y temperatura ambiente. La toma de muestra se hizo directamente sobre los tanques de almacenamiento. El volumen por muestra fue de $5 \mathrm{ml}$, que posteriormente fueron filtradas en papel filtro cualitativo de $125 \mathrm{~mm}$ de diámetro, para seguidamente realizar la medición de hierro total, en el espectrofotómetro de absorción atómica.

Tabla II. Concentración de ácido oxálico goteada a cada pila.

\begin{tabular}{|c|c|}
\hline Pila & Concentración de ácido oxálico [M] \\
\hline 1 & 0.15 \\
\hline 2 & 0.015 \\
\hline 3 & 0.95 \\
\hline
\end{tabular}

Fuente: Autor 


\subsection{Aglomeración}

La aglomeración como pretratamiento sobre el mineral mejora la circulación de la solución a través de la pila (permeabilidad), además evita la segregación de partículas finas entre espacios vacíos dejados por partículas más gruesas, evitando zonas muertas y mejorando la percolación uniforme de la solución a través de la pila [11].

La permeabilidad se ve mejorada gracias a la presencia de partículas de alta densidad como el cuarzo que se depositan en la parte más exterior de los glómeros y las de baja densidad como lo son las arcillas que se depositan en la parte más interior de los glómeros [12]. (Ver Figura 3)

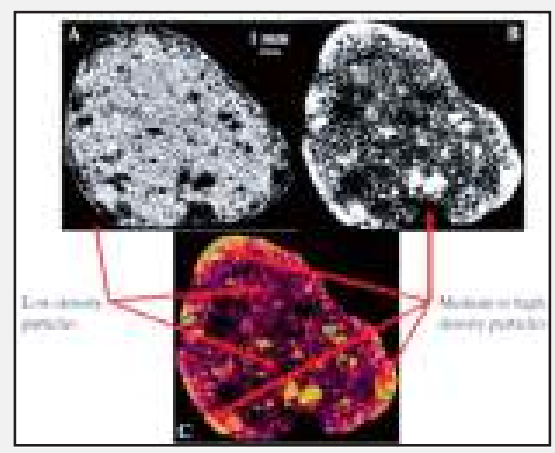

Figura 3. Configuración de estructuras cristalinas como lo son el cuarzo y la arcilla en la configuración de los glómeros Fuente: [12]

\subsection{Caracterización de soluciones de descarga}

Las muestras para su análisis fueron colectadas en diferentes intervalos de tiempo, directamente sobre el tanque almacenamiento. La concentración de hierro total fue determinada usando el espectrofotómetro de absorción atómica (Thermoscientific, Ice $3000^{\circledR}$ ) como se mencionó anteriormente

\section{Resultados y análisis}

\subsection{Caracterización mineralógica}

Los resultados de la Difracción de Rayos X (ver Figura 4) muestran en el mineral un 69\% de Caolinita $\mathrm{Al}_{2} \mathrm{Si}_{2} \mathrm{O}_{5}(\mathrm{OH})_{4}$ y un $31 \%$ de cuarzo $\left(\mathrm{SiO}_{2}\right)$. En la muestra no se reportan los contaminantes del caolín que generan el cambio de la coloración como lo es la Hematita y la goethita, esto se debe a la baja concentración que tienen en el caolín, por eso se realizó una espectrofotometría de absorción atómica, determinando un porcentaje inicial de hierro de $0.19 \%$ en peso.

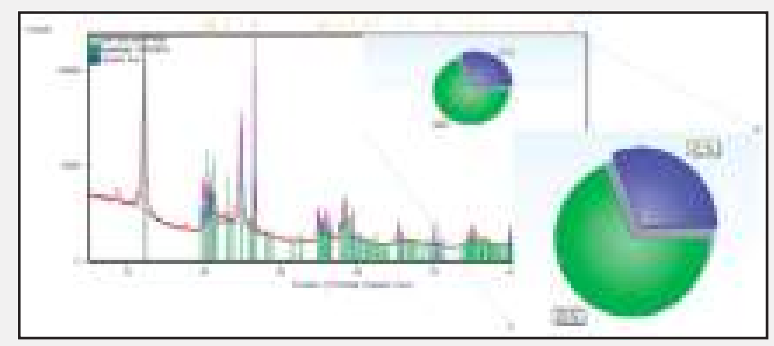

Figura 4. DRX de Caolín antes de blanqueo. Fuente: Autor a partir de ensayo DRX.

En la tabla III se muestran la formula química de cuarzo y caolinita presentes en la muestra de acuerdo con el resultado del ensayo de difracción de Rayos X mencionado anteriormente.

Tabla III. Fases cristalinas del Caolín estudiado.

\begin{tabular}{|c|c|c|}
\hline Ref Code & Compound Name & Chemical Formula \\
\hline $01-086-1560$ & Silicon Oxide & $\mathrm{SiO}_{2}$ \\
\hline $01-083-0971$ & Aluminium Slicate Hydroxide & $\mathrm{Al}_{2}\left(\mathrm{Si}_{2} \mathrm{O}_{5}\right)(\mathrm{OH})_{4}$ \\
\hline
\end{tabular}

Fuente: A partir de ensayo DRX.

\subsection{Blanqueo de Caolín}

\subsubsection{Lixiviación de Hierro}

La lixiviación de hierro se efectuó en tres pilas, sus principales parámetros se muestran a continuación en la Tabla IV. Por nomenclatura en el presente trabajo se van a denominar P1 para la Pila 1, P2 para la Pila 2 y P3 para la Pila 3.
Enero - Junio 2016 ISSN 0122-820X E-ISSN 2422-5053 PP: $65-76$ 
Tabla IV.Variables de operación de las pilas de lixiviación.

\begin{tabular}{|c|c|c|}
\hline PILA 1 & & Valor \\
\hline Variables & Unidades & 30 \\
\hline Altura & Centímetros & 0.15 \\
\hline $\begin{array}{c}\text { Concentración de ácido } \\
\text { Oxálico }\end{array}$ & Molaridad & 3325 \\
\hline Peso de mineral & Gramos & 48.50 \\
\hline Tiempo de lixiviación & Días & 0.19 \\
\hline Hierro Alimento & $\%$ & \\
\hline
\end{tabular}

\begin{tabular}{|c|c|c|}
\hline PILA 2 & & \\
\hline Variables & Unidades & Valor \\
\hline Altura & Centímetros & 30 \\
\hline $\begin{array}{c}\text { Concentración de ácido } \\
\text { Oxálico }\end{array}$ & Molaridad & 0.015 \\
\hline Peso de mineral & Gramos & 3359 \\
\hline Tiempo de lixiviación & Días & 48 \\
\hline Hierro Alimento & $\%$ & 0.19 \\
\hline
\end{tabular}

\begin{tabular}{|c|c|c|}
\hline PILA 3 & & Valor \\
\hline Variables & Unidades & 30 \\
\hline Altura & Centímetros & 0.95 \\
\hline $\begin{array}{c}\text { Concentración de ácido } \\
\text { Oxálico }\end{array}$ & Molaridad & 3265 \\
\hline Peso de mineral & Gramos & 48 \\
\hline Tiempo de lixiviación & Días & 0.19 \\
\hline Hierro Alimento & $\%$ & \\
\hline
\end{tabular}

Fuente: Autor

La Gráfica 1 presenta los resultados de la cinética de la lixiviación de hierro en P1, P2 Y P3 con las diferentes concentraciones de ácidos (0.15M, 0.015M Y 0.95M) en los 49 días de lixiviación.

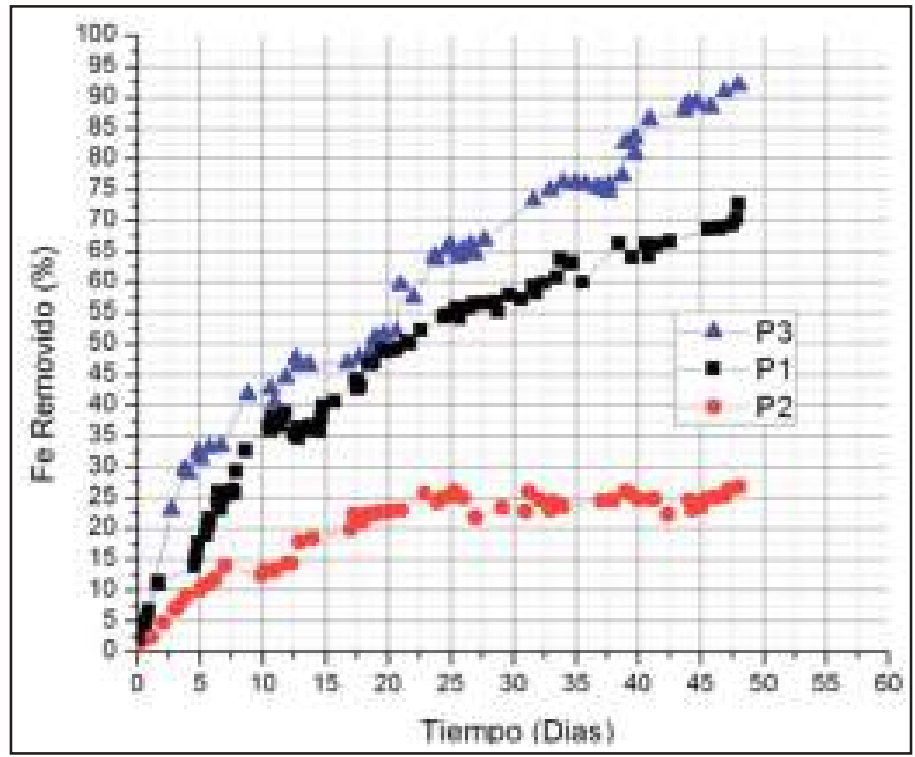

Grafica 1. Cinética de extracción de Fe.

Fuente: Autor 
Observando la Gráfica 1, puede decirse que a medida que aumenta la concentración de ácido oxálico en solución aumenta la remoción de hierro, siendo para P1 Y P3 muy similar hasta los 20 días y para P2 muy notable su baja recuperación por su baja concentración.

Para P1 la recuperación máxima alcanzada fue del $72.2 \%$, para P2 del $26.7 \%$ y para P3 del $90.8 \%$. A medida que el tiempo transcurre, se nota una tendencia exponencial de las 3 pilas, lo que indica que en algún momento la remoción de hierro alcanza el equilibrio, por tal motivo se hace importante modelar y ver en qué momento la derivada de la remoción de hierro con respecto al tiempo se hace cero, para predecir la cantidad máxima de porcentaje de hierro que se va a remover y el tiempo.

\subsubsection{Modelación de la lixiviación de Hierro}

Para cada una de las pilas de caolín se modelo sus comportamientos de remoción de hierro con respecto al tiempo, por esta razón se usó el software Origin ${ }^{\circledR}$, para así determinar el modelo que mejor se ajuste de acuerdo a su $\mathrm{R}^{2}$.

\section{Modelación P1:}

Se ajusta a un modelo exponencial con porcentaje de ajuste de 0.99128 en (6) y Gráfica 2:

$$
\text { Fe Removido }(\%)=70,6729 \mathrm{e}^{\left(-\frac{\text { Dias }}{18,7906}\right)}+72,8967
$$

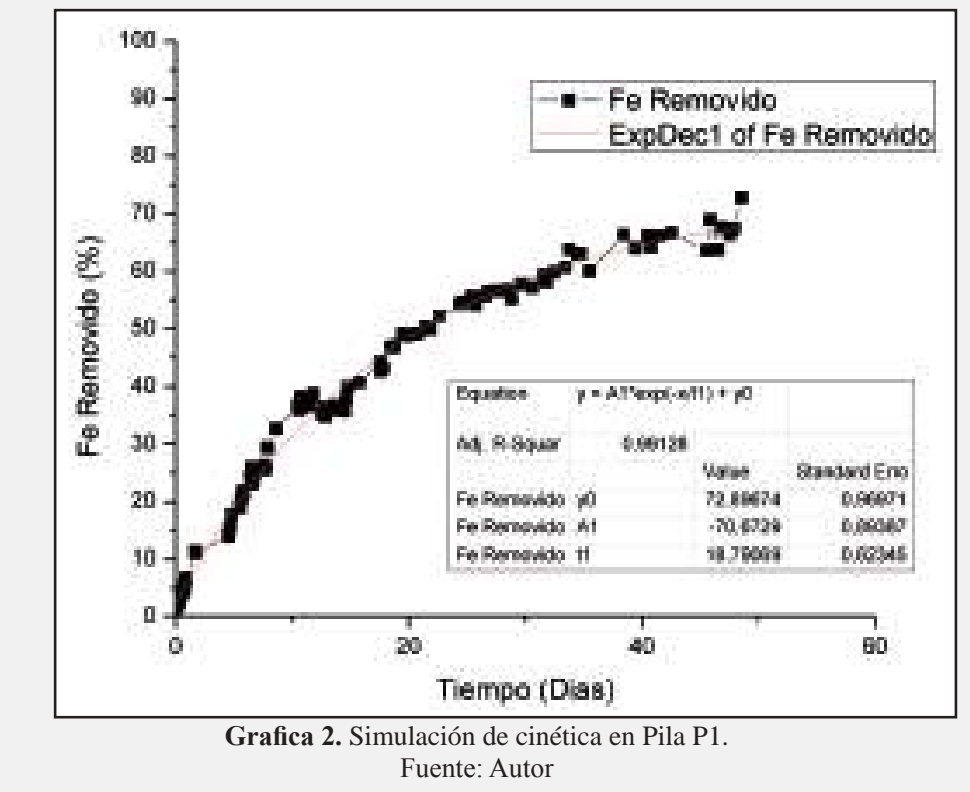

Enero - Junio 2016 ISSN 0122-820X

E-ISSN 2422-5053

A partir de la derivada de la curva cinética, se puede proponer que la remoción máxima en el equilibrio será del $72.55 \%$, en un tiempo de 100 días:
Obteniendo como resultado para P1 en la modelación lo siguiente (ver Tabla V):

Tabla V. Resultados de la simulación para P1.

\begin{tabular}{|c|c|c|}
\hline Fe removido (\%) & Tiempo (Días) & Derivada \\
\hline 73 & 100 & 0,018 \\
\hline \multicolumn{3}{|c}{ Fuente: elaboración propia }
\end{tabular}


Vol. 21

No. 1

Enero - Junio 2016 ISSN 0122-820X E-ISSN 2422-5053 PP: $65-76$

\section{Modelación P2:}

Se ajusta a un modelo exponencial con porcentaje de ajuste 0.95546 en (7) y Gráfica 3:

Fe Removido $(\%)=25,4766 \mathrm{e}^{\left(-\frac{\text { Dias }}{10,6844}\right)}+25,6494$

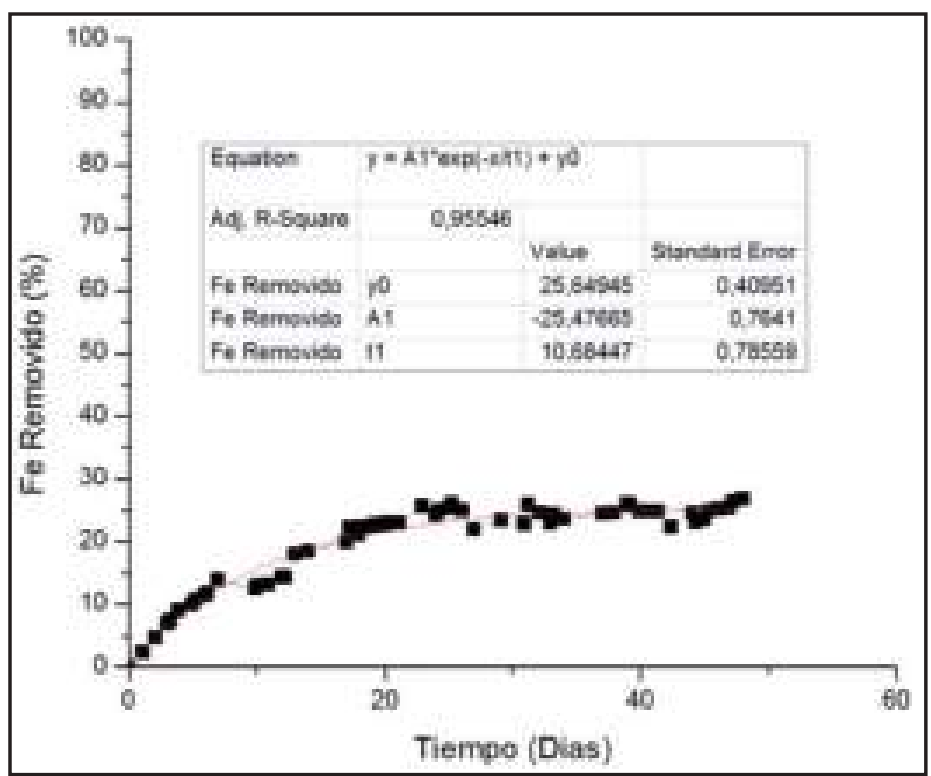

Grafica 3. Simulación de cinética de extracción en P2. Fuente: Autor

Obteniendo como resultado para Pila 2 en la modelación lo siguiente (ver Tabla VI)

Tabla VI. Resultados de la simulación para P2.

\begin{tabular}{|c|c|c|}
\hline Fe removido (\%) & Tiempo (Días) & Derivada \\
\hline 26 & 65 & 0,005 \\
\hline
\end{tabular}

\section{Modelación P3:}

Se ajusta a un modelo exponencial con porcentaje de ajuste de 0.96628 en (8) y Gráfica 4:

$$
\text { Fe Removido }(\%)=115.8 \mathrm{e}^{\left(-\frac{\text { Dias }}{48,6}\right)}+132.8
$$




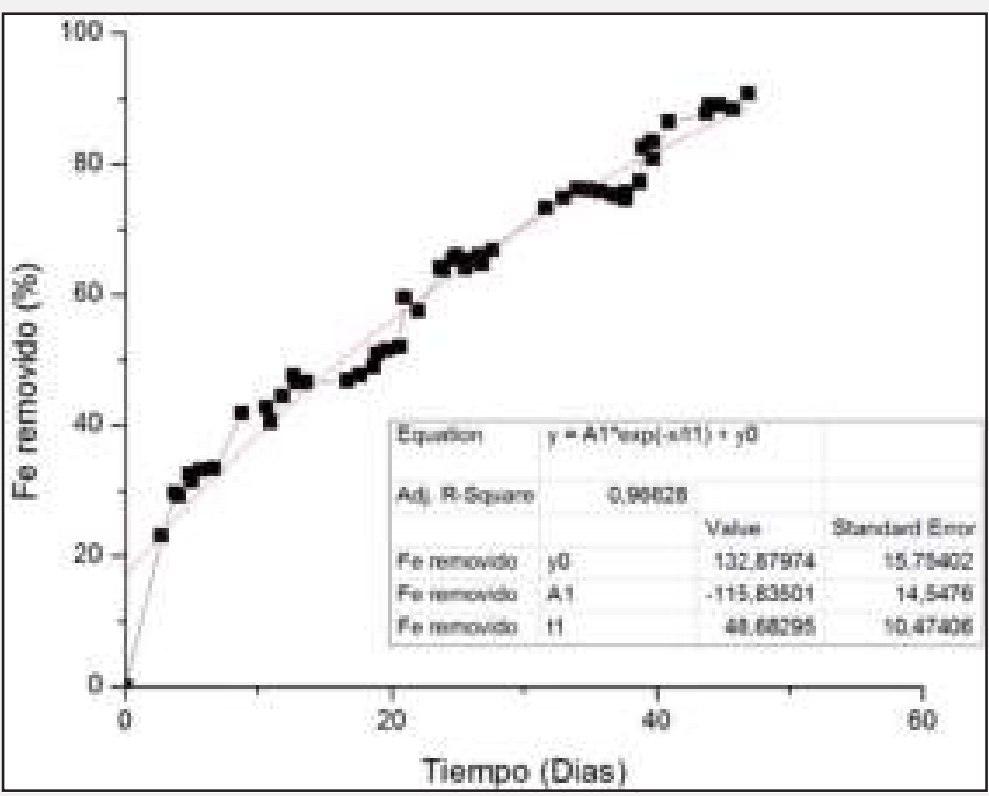

Grafica 4. Simulación de cinética en pila P3.

Fuente: Autor

Obteniendo como resultado para Pila P3 en la modelación lo siguiente (ver Tabla VII)

Tabla VII. Resultados de la simulación para P3.

\begin{tabular}{|c|c|}
\hline Fe removido (\%) & Tiempo (Días) \\
\hline 99 & 62 \\
\hline
\end{tabular}

Fuente: Autor

En general, los porcentajes máximos de hierro removidos a tiempos diferentes para P1, P2 y P3 se muestran a continuación en modo de resumen para ver la relación entre ellas, haciendo énfasis que para P3 se tiene la mayor remoción de hierro en menores días, que P1 y P2 (ver Tabla VIII).

Tabla VIII. Resultados de la simulación para P1, P2 y P3

\begin{tabular}{|c|c|c|}
\hline & $\mathrm{Fe}$ removido (\%) & Tiempo (Días) \\
\hline P1 & 73 & 100 \\
\hline P2 & 26 & 65 \\
\hline P3 & 100 & 63 \\
\hline
\end{tabular}

Fuente: Autor

\subsubsection{Colorimetría}

Se midieron los índices de blancura del mineral antes y después de proceso de lixiviación. Se realizaron tres ensayos de índice de blancura por pila, al material de entrada y salida, a una granulometría de mineral pasante malla 325 (45 micras), debido a que a tamaños inferiores de 45 micras, el caolín tiene sus aplicaciones a nivel industrial.
En la Gráfica 5 y Tabla IX se muestran los índices de blancura alcanzados en las diferentes pilas a diferentes tiempos y donde se evidencia que la pila P3 alcanzó el mayor índice de blancura, debido a la mayor concentración en solución de ácido oxálico. 


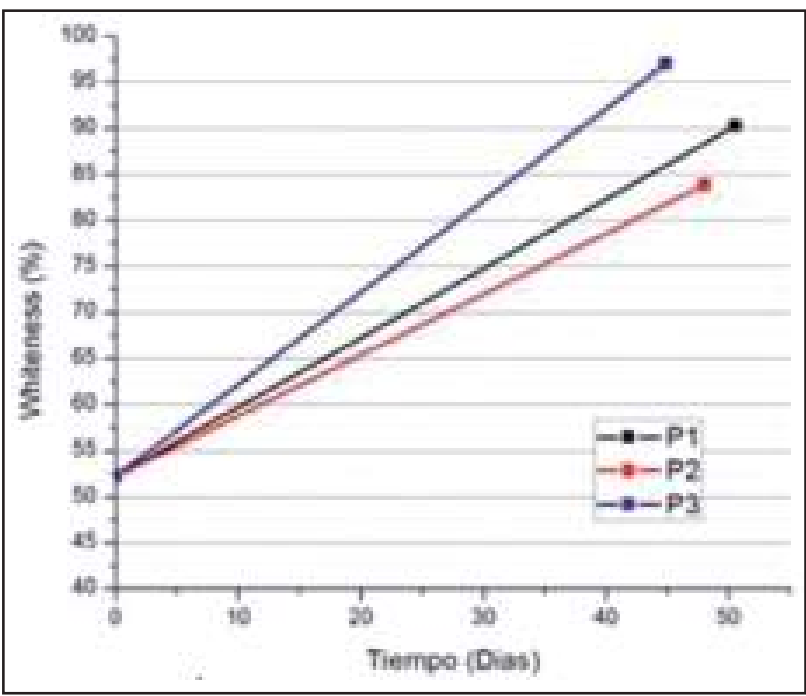

Grafica 5. Índices de blancuras Vs Tiempo, para P1, P2 y P3.

Fuente: Autor

Tabla IX. Ensayos de Índice de blancura para el material de alimento, y el producto final de P1, P2 y P3.

\begin{tabular}{|c|c|c|c|c|}
\hline $\begin{array}{l}\text { Color } \\
\text { Alimento }\end{array}$ & & & & \\
\hline- & Lab* & $\mathbf{a}^{*}$ & $\mathbf{b}^{*}$ & $\% \mathrm{~W}$ \\
\hline Ensayo 1 & 102.80 & -6.17 & 10.11 & 53.97 \\
\hline Ensayo 2 & 103.99 & -8.23 & 8.96 & 52.43 \\
\hline \multirow[t]{2}{*}{ Ensayo 3} & 104.22 & -8.89 & 9.13 & 50.14 \\
\hline & & & $\begin{array}{l}\text { Blancura } \\
\text { definitiva }\end{array}$ & 52.18 \\
\hline \multicolumn{5}{|l|}{ Color } \\
\hline P1 & Lab* & $\mathbf{a}^{*}$ & $\mathbf{b}^{*}$ & $\% \mathrm{~W}$ \\
\hline Ensayo 1 & 102.31 & -5.46 & -1.41 & 90.16 \\
\hline Ensayo 2 & 101.61 & -3.68 & -0.774 & 92.90 \\
\hline \multirow[t]{2}{*}{ Ensayo 3} & 100.51 & -2.17 & 2.13 & 87.63 \\
\hline & & & $\begin{array}{l}\text { Blancura } \\
\text { definitiva }\end{array}$ & 90.23 \\
\hline \multicolumn{5}{|l|}{ Color } \\
\hline P2 & Lab* & $a^{*}$ & $\mathbf{b}^{*}$ & $\% \mathrm{~W}$ \\
\hline Ensayo 1 & 103.32 & -4.67 & 2.04 & 83.19 \\
\hline Ensayo 2 & 102.35 & -3.12 & 1.92 & 87.23 \\
\hline \multirow[t]{2}{*}{ Ensayo 3} & 104.69 & -6.87 & 1.01 & 81.06 \\
\hline & & & $\begin{array}{l}\text { Blancura } \\
\text { definitiva }\end{array}$ & 83.82 \\
\hline \multicolumn{5}{|l|}{ Color } \\
\hline P3 & Lab* & $a^{*}$ & $\mathbf{b}^{*}$ & $\% \mathrm{~W}$ \\
\hline Ensayo 1 & 107.55 & -10.30 & -5.91 & 94.37 \\
\hline Ensayo 2 & 109.46 & -13.22 & -9.98 & 99.73 \\
\hline \multirow[t]{2}{*}{ Ensayo 3} & 109.22 & -13.13 & -9.02 & 96.90 \\
\hline & & & $\begin{array}{l}\text { Blancura } \\
\text { definitiva }\end{array}$ & 97,00 \\
\hline
\end{tabular}

Fuente: Autor

\section{Discusión de resultados}

De acuerdo con la distribución de tamaño de partículas, el $50 \%$ del mineral posee un tamaño inferior a 47.02 micrones, algo que evidencia la alta presencia de finos en las pilas, un factor que reduce severamente la permeabilidad de la pila, pero que a través del proceso de aglomeración fue mitigado. En cuanto espectrofotometría de absorción atómica, se muestra un porcentaje inicial de hierro del $0.19 \%$ en peso del caolín, suficiente para darle coloración roja amarillosa. 
A partir de los resultados experimentales, muestra que a una concentración de ácido oxálico de 0.95 M, se llegó a una remoción de hierro del $90.8 \%$ y un índice de blancura del $97 \%$, seguido por una concentración de ácido oxálico de $0.15 \mathrm{M}$, donde la remoción de hierro fue de $72.2 \%$ y un índice de blancura de $90.23 \%$ y a una concentración de ácido oxálico de $0.015 \mathrm{M}$, una remoción de hierro de $26.7 \%$ y un índice de blancura de $83.82 \%$. Estos resultados expresan la estrecha relación entre la concentración del ácido oxálico y el porcentaje de remoción de hierro de la muestra.

Las modelaciones expresan la máxima remoción posible por cada pila, dando como resultado que a las diferentes concentraciones $0.15 \mathrm{M}, 0.015 \mathrm{M}$ y $0.95 \mathrm{M}$, las máximas remociones fueron del $73 \%, 27 \%$ y $99 \%$ en un tiempo de 100, 65 y 200 días, respectivamente.

\section{Conclusiones}

A mayor concentración de ácido oxálico de goteo, mayor es la remoción de hierro sobre la superficie de caolín, evidenciada por remociones de $72.2 \%, 26.7 \%$ y $90.8 \%$, para concentraciones de ácido $0.15 \mathrm{M}, 0.015 \mathrm{M} \mathrm{Y}$ $0.95 \mathrm{M}$, respectivamente. Es posible hacer lixiviación en pilas con ácido oxálico para el blanqueo de caolín, a pesar de que tiene un contenido muy alto de arcillas, se evidencia que una correcta aglomeración del material evita problemas de conductividad de la solución de ácido oxálico sobre el mineral. La concentración de la solución es una limitante económica, en la Tabla 9 se observa que P3 sólo se necesitaba 63 días para remover el $100 \%$ del hierro soluble, a diferencia de P1 que es de 100 días siendo casi 10 veces mayor la concentración de P3 que la de P1. La presencia de cuarzo y de macro caolines no altera el proceso, dado que no se encontró interferencias con la reacción de reducción del hierro y contrariamente le proporciona estabilidad al glómero, teniendo partículas más gruesas que la de la caolinita. El índice de blancura está directamente relacionado, con el porcentaje de hierro removido sobre la superficie, algo reflejado por los índices de blancuras alcanzados de $90.23 \%$, $83.82 \%$, y $97 \%$, para remociones de hierro de $72.2 \%, 26.7 \%$ y $90.8 \%$, respectivamente en tan sólo 48 días de lixiviación en pila, lo cual es un tiempo corto si compara con la lixiviación de otros minerales como cobre u oro. La velocidad de remoción de hierro es proporcional a la concentración de ácido oxálico goteado. Ya que más iones están dispuestos a reaccionar con la superficie de la Hematita presentes en el caolín. Por lo cual es muy factible y de bajo costo la implementación de este nuevo método de blanqueo de caolín a nivel industrial, por la facilidad del método. Llegando a manejar concentraciones de ácido de $0.15 \mathrm{M}$ en periodos de 50 días.

\section{Agradecimientos}

La investigación expuesta fue realizada en el Instituto de Minerales CIMEX, en patrocinio con COLCIENCIAS, por tal motivo se agradece al instituto por su total colaboración y al proyecto jóvenes investigadores Universidad Nacional de ColombiaCOLCIENCAS 2014.

\section{Referencias}

[1] H. Cornelius, Manual de mineralogia de DANA, Reverte, S. Barcelona, 1978.

[2] Y. $\mathrm{Hu}$ and $\mathrm{X}$. Liu, "Chemical composition and surface property of kaolins," Miner. Eng., no. 11, pp. 12791284, Nov. 2003.

[3] K. Ferguson, "Kaolin utilization in paper and paperboard grows beyond traditional U.S., U.K. Deposits", Pulp \& Paper, vol 77, no. 9, pp. 34, 2003.

ISSN 0122-820X

ISSN 2422-5053

P: 65-76

[4] A. Cabrera La Rosa, "Minerales no metalicos I. Caolin," Ministerio de fomento y obras publicas, Instituto Nacional de Investigación y Fomento Minero. Lima, Peru, 1965. 
Vol. 21

No. 1

Enero - Junio 2016

ISSN 0122-820X

E-ISSN 2422-5053

PP: $65-76$
[5] V. . Ambikadevi y M. Lalithambika, "Effect of organic acids on ferric iron removal from iron-stained kaolinite", Applied Clay Science, vol. 16, no. 3-4, pp. 133-145, Mar. 2000.

[6] A. M. Gómez Jiménez y M. O. Bustamante Rua, "Estudio del efecto de la fracción de ultrafinos, sobre blanqueo de caolines". Tesis de Maestría, Universidad Nacional de Colombia, Medellín, Colombia, 2012.

[7] A. M. Muñoz García and M. O. Bustamante Rua, "Blanqueo de caolines de la union Antioquia usando metodos hidro-electrometalúrgicos". Tesis de Maestría, Universidad Nacional de Colombia, Medellín, Colombia, 2010.

[8] D. Panias, M. Taxiarchou, I. Douni, I. Paspaliaris, y A. Kontopoulos, "Mechanisms of dissolution of iron oxides in aqueous oxalic acid solutions", Hydrometallurgy ,vol. 42, no. 2, pp.257-265, 1996.

[9] J. P. Pinzón Forero, "Balance de masa en la planta de blanqueo de Caolín - SUMICOL". Tesis de pregrado, Universidad Nacional de Colombia, Medellín, Colombia, 2010.

[10] M. L. Free. Hidrometallurgy: Fundamentals and applications. John Wiley \& Sons, Inc., Hoboken, New Jersey, 2013.

[11] N. Dhawan, M. S. Safarzadeh, J. D. Miller, M. S. Moats, y R. K. Rajamani, "Crushed ore agglomeration and its control for heap leach operations" Minerals Engineering, no. 41, pp. 5370, Feb. 2013.

[12] A. Nosrati, W. Skinner, D. J. Robinson, y J. Addai-Mensah, "Microstructure analysis of $\mathrm{Ni}$ laterite agglomerates for enhanced heap leaching", Powder
Technology, vol. 232, pp. 106-112, Dec. 2012. 